\title{
MedDRA High Level Term
}

National Cancer Institute

\section{Source}

National Cancer Institute. MedDRA High Level Term. NCI Thesaurus. Code C71880.

A superordinate descriptor for one or more MedDRA preferred terms (PTs). It is subordinate to high level group term (HLGT). 